\title{
Supporting Learning Analytics in Computing Education
}

\author{
Daniel M. Olivares \\ Human-Centered Environments for Learning and \\ Programming (HELP) Lab \\ School of Electrical Engineering and Computer Science \\ Washington State University \\ Pullman, WA 99164 USA \\ daniel.olivares@wsu.edu
}

\author{
Christopher D. Hundhausen \\ Human-Centered Environments for Learning and \\ Programming (HELP) Lab \\ School of Electrical Engineering and Computer Science \\ Washington State University \\ Pullman, WA 99164 USA \\ hundhaus@wsu.edu
}

\begin{abstract}
As is the case for many undergraduate STEM degree programs, computing degree programs are plagued by high attrition rates. This is especially true in early computing courses, in which failure and drop-out rates in the 35 to 50 percent range are common. By collecting learning process data as students engage in computer programming assignments, computing educators can place themselves in a position not only to better understand students' struggles, but also to better tailor instructional interventions to students' needs. We have developed OSBLE+, a learning management and analytics environment that interfaces with a computer programming environment to support the automatic collection of learners' programming process and social data as they work on programming assignments, while also providing an interactive environment for the analysis and visualization of those data. In ongoing work, we are using OSBLE+ to explore two possibilities: (a) leveraging learning and social data to strategically deliver automated learning interventions, and (b) presenting learners with visual representations of their learning data in order to prompt them to reflect on and discuss their learning processes.
\end{abstract}

\section{CCS Concepts}

- Social and professional topics Computing education

- Applied computing Learning management systems

\section{Keywords}

Computing education research; Learning analytics; Learning Management System; Social Learning; Data collection; Visualizations; OSBLE

\section{INTRODUCTION}

STEM undergraduate degree programs are notorious for having high drop-out and failure rates. Computing degree programs are among the worst culprits; attrition rates of 35 to 50 percent are common, especially in early courses. Learning analytics constitutes a promising way to address this problem: If we can capture learning process data as students work on computer programming assignments, we can use those data to understand their struggles, and ultimately to better tailor instruction to their needs.

To that end, we have been developing OSBLE+, a learning management and analytics environment that facilitates the collection and analysis of learning process data in computing courses (see Figure 1). These data include not only programming process data such as compilations, edits, and run-time exceptions,

Permission to make digital or hard copies of part or all of this work for personal or classroom use is granted without fee provided that copies are not made or distributed for profit or commercial advantage and that copies bear this notice and the full citation on the first page. Copyrights for thirdparty components of this work must be honored. For all other uses, contact the Owner/Author.

Copyright is held by the owner/author(s).

$L A K^{\prime} 17$, March 13-17, 2017, Vancouver, BC, Canada

ACM 978-1-4503-4870-6/17/03.

http://dx.doi.org/10.1145/3027385.3029472 but also social data: students' asynchronous discussions as they engage in computer programming. In this poster, we will present the design of OSBLE + , including its software architecture and user interface, and we will highlight our ongoing efforts to further develop OSBLE + and explore its ability to help facilitate improved learning processes and outcomes in computing education.

\section{RELATED WORK}

Within computing education, initial interest in learning analytics was spurred by an interest in building predictive models of learning success. Many such models (e.g., [2, 4, 8]) were based on programming process data collected within an integrated development environment (IDE), where students typically spend large amounts of out-of-class time working on individual programming assignments. An outgrowth of one of these [4] is the Black Box project [1], which makes publicly-available a large corpus of data automatically collected through the BlueJ novice programming environment [6].

While Black Box makes programming process data available, it does not provide a means of analyzing the data. By supporting the interactive visual exploration of learning process data, learning analytics dashboards (e.g., see [7]) provide one such means. They can be used to augment face-to-face teaching, online learning, or even blended learning settings [7]. They can be part of LMSs such as Moodle, custom LMSs as seen in [5], or a student centered dashboard that uses of both learning analytics and formative assessment [5]. OSBLE+ contributes to this line of research through its development of a learning analytics dashboard for computing instructors.

\section{OSBLE + DESIGN}

Figure 2 presents an overview of the OSBLE+ software architecture, which includes three key components:

- The Visual Studio integrated development environment (IDE) is where computing students spend much of their out-of-class time

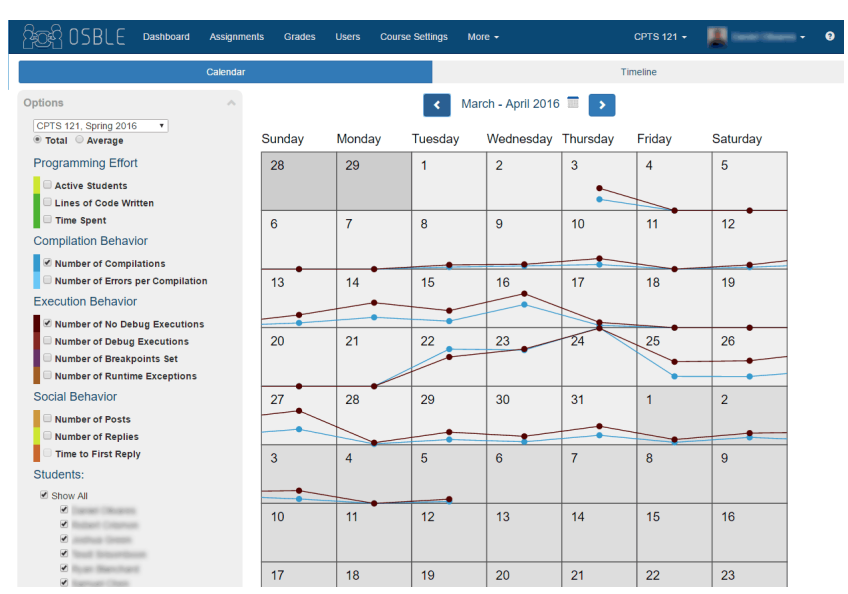

Figure 1. The OSBLE+ learning analytics dashboard 


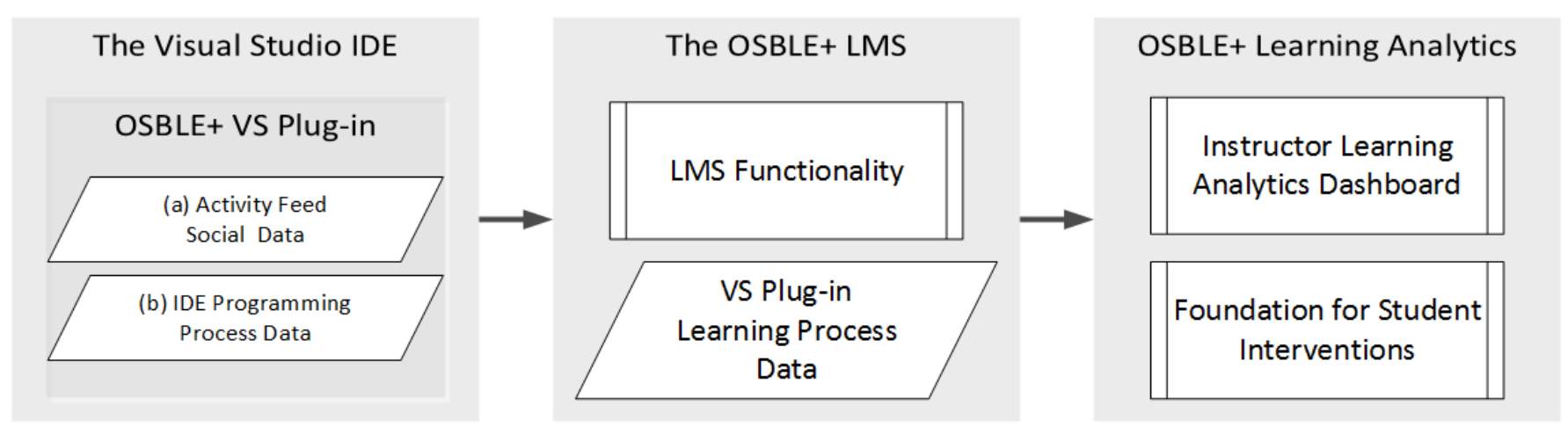

Figure 2. An overview of the OSBLE+ software architecture

working on individual computer programming assignments, which are typically the centerpiece of computing courses.

- The OSBLE+ plug-in to the Visual Studio IDE serves two key purposes: (a) it augments Visual Studio with an activity stream, which provides an asynchronous forum for students to discuss issues that arise during the programming process (see Figure 3); and (b) it automatically collects students' programming process data (e.g., edits, compilation attempts, run-time exceptions) and social data (activity stream posts and replies).

- The OSBLE+ learning management system (LMS) provides many traditional LMS functions, such as a file system for disseminating course materials, and an assignment system for posting and submitting course assignments. However, because it receives learning process data from the OSBLE+ plug-in, it can also present those data to learners and instructors. In the present implementation, OSBLE+ provides the instructor with a learning analytics environment for exploring student learning process data Within this environment, instructors can select a set of data to visualize, along with a range of dates, and then see line graphs of those data within a calendar view (see Figure 1).

\section{STATUS AND FUTURE WORK}

To date, we have used various versions of OSBLE+ in large CS1 and CS2 courses at Washington State University. .In an empirical study of the CS2 course, student social participation was strongly correlated with assignment success [3]. Motivated by this result and by social learning theory, we are presently exploring ways to use students' programming and social data as a basis for automated interventions-delivered directly within the IDE-that nudge

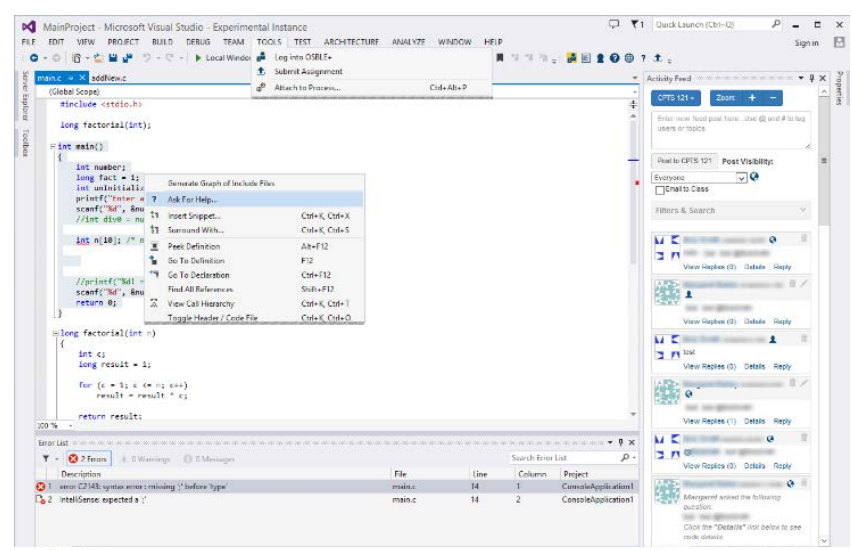

Figure 3. The Visual Studio IDE with the OSBLE+ plug-in students toward greater social participation at strategic points in the learning process. We are also interested in injecting visual representations of learner data into the activity stream, in order to prompt students to engage in reflective conversations about their learning processes.

\section{ACKNOWLEDGMENTS}

This project is funded by the National Science Foundation under grant no IIS-1321045

\section{REFERENCES}

[1] Brown, N.C.C. et al. 2014. Blackbox: A Large Scale Repository of Novice Programmers' Activity. Proceedings of the 45th ACM Technical Symposium on Computer Science Education. ACM. 223-228.

[2] Carter, A.S. et al. 2015. The Normalized Programming State Model: Predicting Student Performance in Computing Courses Based on Programming Behavior. Proceedings of the Eleventh Annual International Conference on International Computing Education Research. ACM. 141-150.

[3] Carter, A.S. and Hundhausen, C.D. 2016. With a Little Help From My Friends: An Empirical Study of the Interplay of Students' Social Activities, Programming Activities, and Course Success. Proceedings of the 2016 ACM Conference on International Computing Education Research. ACM. 201209.

[4] Jadud, M.C. 2006. Methods and Tools for Exploring Novice Compilation Behaviour. Proceedings of the Second International Workshop on Computing Education Research. ACM. 73-84.

[5] Rößling, G. et al. 2008. Enhancing Learning Management Systems to Better Support Computer Science Education. SIGCSE Bull. 40, 4 (Nov. 2008), 142-166.

[6] Utting, I. et al. 2012. Web-scale Data Gathering with BlueJ. Proceedings of the Ninth Annual International Conference on International Computing Education Research. ACM. 1-4.

[7] Verbert, K. et al. 2014. Learning Dashboards: An Overview and Future Research Opportunities. Personal Ubiquitous Comput. 18, 6 (Aug. 2014), 1499-1514.

[8] Watson, C. et al. 2014. No Tests Required: Comparing Traditional and Dynamic Predictors of Programming Success. Proceedings of the 45th ACM Technical Symposium on Computer Science Education. ACM. 469-474. 